\title{
Marked eosinophilia due to intestinal strongyloidiasis in an immunocompetent patient
}

\author{
K M D J Rodrigo', R Premaratna², T G A N Chandrasena', N R de Silva', H J de Silva ${ }^{2}$
}

Ceylon Medical Journal 2012; 57: 172-173

\section{Introduction}

Strongyloides stercoralis is unique among the intestinal nematodes in that it has a free-living cycle in warm and tropical climates in addition to a parasitic cycle in its human host. In immuno-competent hosts, this parasite is mostly confined to the intestinal tract. Infection may be asymptomatic or it may induce a wide variety of symptoms such as nausea, vomiting, abdominal pain, chronic diarrhea, loss of weight and pruritus of the perianal region caused by autoinfective larvae ('larva currens'). Although intestinal nematode infections are relatively common in Sri Lanka, infection with S. stercoralis is infrequently reported. Prevalence rates of $S$. stercoralis infection in Sri Lanka range from 0-1.6\% [1,2,3]. Here we report a case of symptomatic intestinal strongyloidiasis with marked eosinophilia.

\section{Case report}

A 47-year old previously healthy male was admitted with a history of diarrhoea and right-sided abdominal pain of six weeks duration. He complained of passing watery stools 5-6 times a day which was not associated with passage of blood or mucus. There was no steatorrhoea. Initially, loose motions were associated with nausea and vomiting and symptoms were more prominent following meals. He had lost about $10 \mathrm{~kg}$ in weight during this period, and also experienced significant loss of appetite. He also complained of intermittent low grade fever without night sweats. There was no history of chronic cough or wheeze. On examination he was not pale or icteric and there was no lymphadenopathy. There was mild clubbing. No peripheral oedema was noted. He had a chronic eczematous skin rash in both lower limbs and significant excoriation of the perianal skin suggestive of larva currens. Examination of the cardiovascular and respiratory systems was unremarkable. The only physical sign in the abdomen was tenderness in the right lower quadrant.

His total leucocyte count was $47.7 \times 10^{3} / \mu 1$ with an eosinophil count of $80 \%$. Haemoglobin was $13.5 \mathrm{~g} / \mathrm{dl}$ and the platelet count was $213 \times 10^{3} / \mu 1$. His blood picture showed marked eosinophilia with normal morphology. His ESR was $42 \mathrm{~mm} / 1 \mathrm{st}$ hour and C-reactive protein was 3.1 $\mathrm{mg} / \mathrm{dl}$. Renal and liver function tests were within normal limits except for mild hypoalbuminaemia. Chest X-ray and ultrasound abdomen were normal. Microscopic examination of a saline smear from a faecal sample for parasites demonstrated numerous rhabditiform larvae which were presumptively identified as those of $S$. stercoralis by their short buccal capsule and prominent genital primordium (Figure 1).

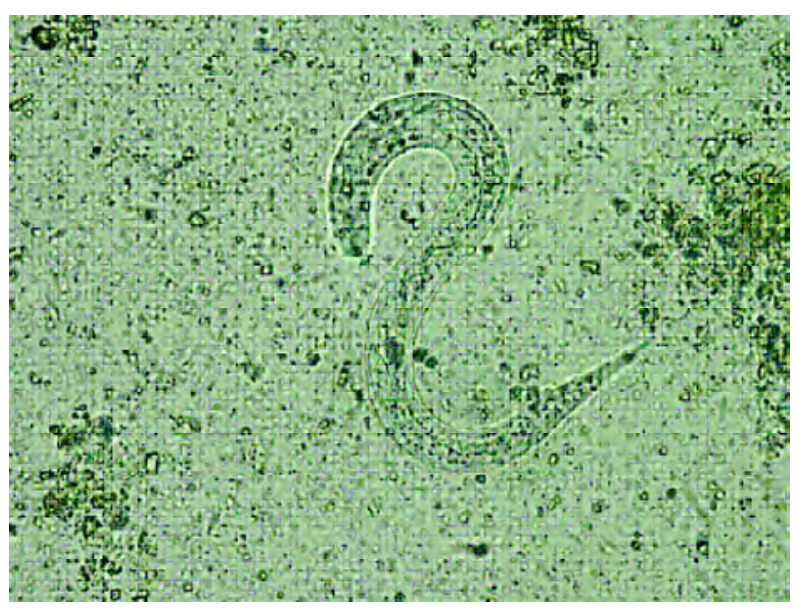

Figure 1. L1 rhabditiform larva of Strongyloides stercoralis in direct faecal smear.

Coprocultures performed on charcoal, on nutrient agar, and using the Harada-Mori technique, yielded $\mathrm{L}_{3}$ filariform larvae which confirmed the species identification by its long oesophagus and the tail with a notched tip (Figure 2).

A colonoscopy was performed to exclude any other associated pathology and was macroscopically normal. Series of colonic and ileal biopsy histology revealed eosinophilic gastroenteritis with lamina propria being heavily infiltrated with eosinophils admixed with lymphocytes and neutrophils and some crypt abscesses (Figure 3). He had no history of recurrent infections in the past. His serum globulin level was within normal limits and quantitative serum immunoglobulin levels were not done. The retroviral screen was negative.

Departments of ${ }^{1}$ Parasitology, ${ }^{2}$ Medicine, Faculty of Medicine, University of Kelaniya, Sri Lanka.

Correspondence: KMDJR, e-mail: <damith_rodrigo@yahoo.com>. Received 10 May and revised version accepted 8 July 2012. Competing interests: none declared. 

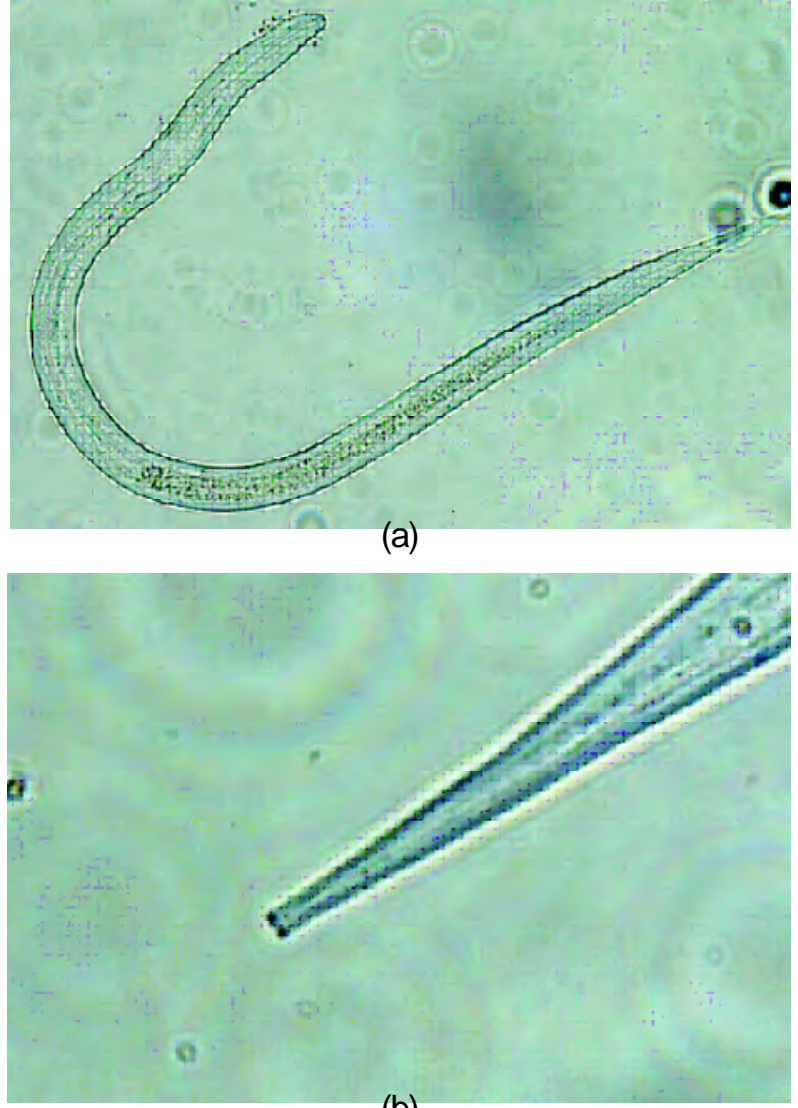

(b)

Figure 2. (a). $L_{3}$ filariform larva of Strongyloides stercoralis in faecal culture, (b). Notched tail of $\mathrm{L}_{3}$ larva.

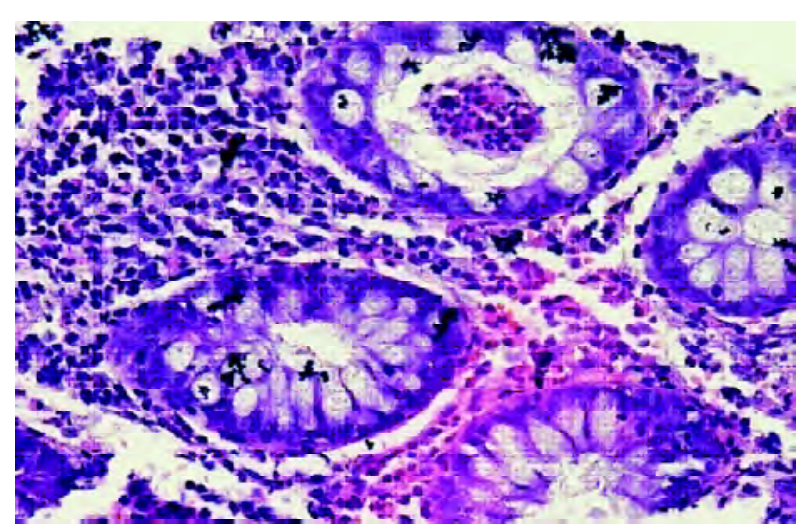

Figure 3. Colonic biopsy showing eosinophilic gastroenteritis with a crypt abscess (white arrow).

A diagnosis of intestinal strongyloidiasis was confirmed and treatment was initiated with the antihelmintic albendazole at a dosage of $400 \mathrm{mg}$ twice daily for 7 days, since ivermectin is not registered for human use in Sri Lanka. His abdominal symptoms improved and bowel habits became normal. Faecal examination was repeated after completion of the first antihelmintic course, and revealed persistent infection (rhabditiform larvae in direct faecal smears and $\mathrm{L}_{3}$ filariform larvae in stool cultures). Therefore, albendazole was repeated at the same dosage.
Direct faecal microscopy following the second course of albendazole was negative for rhabditiform larvae, but the faecal cultures continued to yield $\mathrm{L}_{3}$ filariform larvae, indicating persistent low grade infection. However, following the third course of high dose albendazole the infection was cleared.

\section{Discussion}

The most distinct feature of S. stercoralis is its ability to replicate within the host. Even in immuno-competent cases, a small population of adult worms remains alive and propagates in the small intestines for decades without further infection from outside [4]. This causes recurrent clinical manifestations, which may include skin rashes at the sites where the filariform larvae enter the perianal skin ('larva currens' associated with urticaria) similar to that observed in our patient.

The definitive diagnosis of strongyloidiasis is made on the basis of detection of larvae in faeces. Direct faecal smears are easy and quick to perform [5]. Faecal culture techniques (Harada-Mori filter paper, charcoal cultures and agar plate cultures) which harvest the $\mathrm{L}_{3}$ larvae from faeces are more labour intensive but have a higher sensitivity than direct faecal smears and are useful in differentiating $S$. stercoralis infection from other intestinal nematode infections [6,7]. This case of intestinal strongyloidiasis highlights the importance of careful follow-up of cases after treatment, even in immunocompetent individuals, until final parasitological clearance.

\section{Acknowledgements}

We thank Mr. H. Sudusinghe and Mr. Nilaweera for technical assistance.

\section{References}

1. Sorensen E, Ismail M, Amarasinghe DKC, Hettiarachchi I, Dassanaike TS. The prevalence and control of soil transmitted nematode infections among children and women in the plantations in Sri Lanka. Ceylon Medical Journal 1996; 41: $37-41$.

2. Ramadas G, Ramadas D. Patterns of parasites in a paediatric ward in the General Hospital (Teaching) Jaffna. Ceylon Journal of Child Health 1989; 18: 29-33.

3. Nageswaran C, Sivarajah N. Intestinal parasitic infestations in children living in the under privileged sector of the Jaffna municipality. Jaffna Medical Journal 1986; 21: 23-8.

4. Lawrence LP, Tesfai G, 1985. Chronic strongyloidiasis in Vietnam veterans. American Journal Medicine 1985; 78: 139-40.

5. Weller PF, Leder KL. Strongyloidiasis. Available at http:// www.uptodate.com/contents/strongyloidiasis.

6. De Kaminsky RG. Evaluation of three methods for labouratory diagnosis of Strongyloides stercoralis infection. Journal of Parasitology 1993; 79: 277-80.

7. Morel R, Ekanayake S, Abeykoon S. Strongyloides stercoralis isolated by agar plate culture. Ceylon Medical Journal 2007; 52: 150. 